\title{
Diversidad de Hemiptera asociada a árboles de ambientes urbanos de la provincia de Jujuy, Argentina
}

\author{
Verónica C. Hamity ${ }^{12}$; Eugenia F. Contreras ${ }^{1}$ \& Javier Altamirano ${ }^{3, \bigotimes}$ \\ ${ }^{1}$ Instituto de Biología de la Altura (INBIAL), Universidad Nacional de Jujuy, San Salvador de Jujuy, Argentina. ${ }^{2}$ Facultad \\ de Ciencias Agrarias, Universidad Nacional de Jujuy. San Salvador de Jujuy, Argentina. ${ }^{3}$ Instituto de Ecorregiones Andinas \\ (INECOA), UNJu-CONICET, Instituto de Biología de la Altura, San Salvador de Jujuy, Argentina.
}

\begin{abstract}
Resumen. Los Hemiptera de ambientes urbanos de Jujuy son poco conocidos y explorados. El objetivo de este trabajo fue determinar y caracterizar la diversidad de Hemiptera asociada a tres especies del arbolado público de Jujuy, y aportar datos bioecológicos que contribuyan a su conocimiento. El estudio se realizó en tres ciudades durante una estación seca y una húmeda, y para cada localidad se consideraron dos ambientes: urbano y suburbano. En cada sitio se muestrearon tres árboles de Handroantus impetiginosus, Enterolobium contortisiliquum y Citrus aurantium. Se aspiró el follaje y se extrajeron al azar 10 ramas/árbol. Se identificaron los hemípteros y se comparó la abundancia, la riqueza, la diversidad, la composición y la dominancia de cada suborden en función de las especies arbóreas, las localidades, la estacionalidad y el grado de urbanización. Colectamos 4339 individuos pertenecientes a los subórdenes Auchenorrhyncha ( 8 familias, 30 morfoespecies, 1044 individuos), Heteroptera (8 familias, 20 morfoespecies, 196 individuos) y Sternorrhyncha (9 familias, 33 morfoespecies, 3099 individuos). Por primera vez para Jujuy se cita a Rhabdotalebra albinoi y a Rhabdotalebra flava sobre E. contortisiliquum, y a $R$. flava y al género Tingis sobre H. impetiginosus. La localidad con mayor riqueza fue San Salvador de Jujuy (56 morfoespecies, 28 exclusivas), en contraste con El Carmen (39 morfoespecies, 13 exclusivas) y San Pedro (34 morfoespecies, 11 exclusivas). Registramos heterópteros fitófagos, depredadores y de dieta mixta. Sternorrhyncha fue el suborden mejor representado en riqueza y abundancia teniendo en cuenta localidad y especie arbórea, mientras que la estacionalidad no lo afectó como a Auchenorrhyncha y Heteroptera. No encontramos diferencias en abundancia entre el sector urbano y el suburbano. Las especies de hemípteros compartidas por las tres especies de árboles fueron Dikrella sp., Empoasca sp.2, Aleyrodidae sp.1 y Pseudococcidae sp.1; los dominantes en lapacho fueron Rabdotalebra ava, Rabdotalebra sp.2, Tingis sp.1 y Ceroplastes sp.1; en naranjo, Lephidosaphes beckii, Aphididae sp.1 y Aonidiella aurantii; en pacará, Psyllidae sp.2, Aphididae sp.1 y Empoasca sp.2.
\end{abstract}

[Palabras clave: bioecología, Auchenorrhyncha, Sternorrhyncha, Heteroptera, lapacho, naranjo, pacará, arbolado público]

\begin{abstract}
Aвstract. Hemiptera diversity associated with trees from urban environments in the province of Jujuy, Argentina. The Hemiptera of urban environments in Jujuy are little known and quite unexplored. The objective of this work was to analyze and characterize the Hemiptera diversity in trees of public roads in Jujuy, and to provide data about their bioecology. The study was conducted in three locations during a dry and a wet season; two environments were considered for each location: urban and suburban. In each site, three trees of Handroantus impetiginosus, Enterolobium contortisiliquum and Citrus aurantium were sampled. The foliage was aspirated and 10 branches/tree were randomly extracted. We identified the hemipterans and compared the abundance, richness, diversity, composition and dominance of each suborder according to the tree species, localities, seasonality and degree of urbanization. We collected 4339 individuals belonging to the suborders Auchenorrhyncha ( 8 families, 30 morphospecies, 1044 individuals), Heteroptera ( 8 families, 20 morphospecies, 196 individuals) and Sternorrhyncha (9 families, 33 morphospecies, 3099 individuals). For the first time in Jujuy, Rhabdotalebra albinoi and Rhabdotalebra flava were recorded on E. contortisiliquum, and Rhabdotalebra flava and the genus Tingis on H. impetiginosus. San Salvador de Jujuy was the location with the highest richness (56 morphospecies, 28 exclusive), in contrast to El Carmen (39 morphospecies, 13 exclusive) and San Pedro (34 morphospecies, 11 exclusive). We recorded Heteroptera phytophages, predators and species with mixed diet. Considering locality and tree species, Sternorrhyncha was the suborder with highest richness and abundance, whereas seasonality did not affect it. The other two suborders were not affected by seasonality. Hemiptera species shared by the three tree species were Dikrella sp., Empoasca sp.2, Aleyrodidae sp.1 and Pseudococcidae sp.1. The dominant species in lapacho were Rabdotalebra flava, Rabdotalebra sp.2, Tingis sp.1 and Ceroplastes sp.1; in orange, Lephidosaphes beckii, Aphididae sp.1 and Aonidiella aurantii; in pacará, Psyllidae sp.2, Aphididae sp.1 and Empoasca sp.2.
\end{abstract}

[Keywords: bioecological, Auchenorrhyncha, Sternorrhyncha, Heteroptera, lapacho, orange tree, pacará, public woodland]

Editora asociada: Adriana Salvo
Recibido: 3 de Abril de 2019

Aceptado: 20 de Agosto de 2019 


\section{INTRODUCCIÓN}

Las ciudades se caracterizan por presentar una amplia variedad de condiciones ambientales como resultado de la intervención antrópica. Entre estas condiciones sobresale la creación de parches heterogéneos compuestos por diferentes tipos de edificaciones y zonas verdes, que pueden sustentar una particular y rica diversidad biológica (Rebele 1994; Mc Kinney 2002). Los artrópodos, y en especial los insectos, dominantes por su riqueza, abundancia y biomasa en los ecosistemas terrestres (Erwin 1982; Wilson 1985; Stork 1988; Gaston 1991), constituyen un grupo aún poco conocido en los escenarios urbanos (McIntyre 2000).

Entre los insectos exopterigotas hemimetábolos, los Hemiptera representan el grupo dominante y más grande en cuanto a su diversidad y abundancia (Carver et al. 1991; Grazia et al. 2012). Entre otros caracteres, se los puede reconocer por la estructura particular de su aparato bucal, adaptado para picar y succionar, característica diagnóstica de este orden (Carver et al. 1991). Sus hábitos alimentarios varían de acuerdo con el suborden al que pertenecen; los Auchenorrhyncha y Sternorrhyncha son estrictamente fitófagos, con posibilidad de ser vectores de enfermedades, y los Heteroptera, además de fitófagos pueden ser depredadores, hematófagos y ectoparásitos (Schuh and Slater 1995).

Hasta el presente, la fauna de Hemiptera en la provincia de Jujuy fue explorada principalmente en cultivos agrícolas y plantas ornamentales, y por su interés en medicina (Agostini de Manero and Muruaga De L'Argentier 1987; Muruaga De L'Argentier and Agostini 1995; Morrone and Coscarón 1998; Linares and Neder 1999; Claps et al. 2008; Linares et al. 2010; Contreras and Coscarón 2012; Catalano et al. 2013a, b y c; Melo and Dellapé 2013; Roig-Juñent et al. 2014; Dellapé 2015; Dellapé et al. 2015; Dellapé et al. 2016; Hamity et al. 2016; Coscarón 2017). La riqueza específica de las comunidades naturales en ambientes urbanos y suburbanos aún permanece desconocida.

La provincia de Jujuy tiene una variedad de ecorregiones que van desde zonas áridas y semiáridas hasta húmedas y selváticas, zonas de transición y núcleos de urbanización, que junto con un gradiente altitudinal favorecen su diversidad florística y faunística. Esto genera diferencias en la complejidad de las comunidades animales (Cabrera 1976; Brown et al. 2005). En los valles y el ramal de Jujuy, las ciudades se caracterizan por estar rodeadas por cerros con vegetación natural y circundadas por ríos y arroyos con presencia de numerosas especies de insectos que suelen ser frecuentes en ambientes silvestres. Como parte de los parches verdes de las ciudades, el arbolado público representa un abanico de posibilidades para que los insectos, y entre ellos los hemípteros, puedan residir, alimentarse y reproducirse.

Entre las especies arbóreas nativas del arbolado público de las regiones del valle y ramal de la provincia de Jujuy se destacan Tipuana tipu (Benth) Kuntze (tipa), Handroantus albus (Cham.) Mattos (lapacho amarillo), Handroantus impetiginosus (Mart. Ex D.C.) Standl. (lapacho rosado), Erythrina crista-galli L. (ceibo), Jacaranda mimosifolia, D. Don (jacarandá), Enterolobium contortisiliquum (Vell.) Morong (pacará), Ceiba speciosa (A. StHill) Ravenna (palo borracho rosado) y Ceiba insignis (Kunth) P. E. Gibbs and Semir (palo borracho amarillo). Entre de las especies introducidas se encuentran Morus alba Linn. (mora blanca), Morus nigra L. (mora negra), Citrusaurantium L. (naranjo), Fraxinusamericana L. (fresno blanco), Magnolia grandiflora, L. (magnolia), Grevillea robusta Cunn ex R. Br. (roble sedoso), Platanus orientalis L. (plátano), Populus nigra, L. (álamo), Populus alba, L. (álamo plateado), Tilia americana L. (tilo), Ulmus spp Mill. (olmo), Lagerstroemia indica (L.) Pers. (crespón) y Persea americana Mill. (palto) (Biloni 1990; Romeo and Entrocassi 2012).

El objetivo de este trabajo fue determinar y caracterizar la diversidad de Hemiptera asociada a tres especies del arbolado público de tres localidades de la provincia de Jujuy en ambientes urbanos y suburbanos. Además, se buscó aportar datos bioecológicos que contribuyan al conocimiento de la fauna estudiada.

\section{MAteriales y MÉTOdos}

El estudio se realizó en tres localidades de la provincia de Jujuy, Argentina: San Pedro

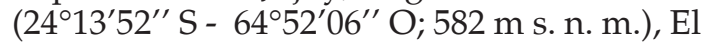
Carmen (24 $23^{\prime} 21^{\prime \prime} \mathrm{S}-6^{\circ} 15^{\prime} 42^{\prime \prime} \mathrm{O} ; 1179 \mathrm{~m} \mathrm{~s}$. n. m.) y San Salvador de Jujuy $\left(24^{\circ} 11^{\prime} 09^{\prime \prime} \mathrm{S}\right.$ $65^{\circ} 17^{\prime} 58^{\prime \prime} \mathrm{O} ; 1272 \mathrm{~m} \mathrm{s.n.m}$.) (Figura 1), durante septiembre-octubre de 2016 (estación seca) y febrero-marzo de 2017 (estación húmeda). Los muestreos se realizaron sobre las especies 


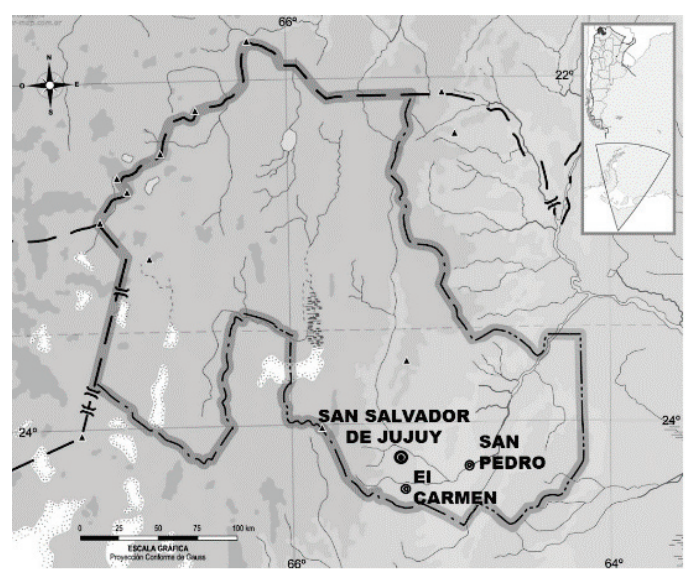

Figura 1. Ubicación de los sitios de estudio en la provincia de Jujuy.

Figure 1. Location of the study sites in the province of Jujuy.

arbóreas $H$. impetiginosus (lapacho rosado), E. contortisiliquum (pacará) y C. aurantium (naranjo) por estar presentes y ser comunes en las tres localidades estudiadas.

El pacará y el lapacho son especies nativas que presentan una amplia distribución en el Noroeste Argentino (NOA). Se los encuentra entre los 600 y 1200 m s. n. m., en el piso inferior de las Yugas (selva pedemontana) y en la transición con el Chaco Serrano. Por sus características morfológicas, biológicas, porte y floración son especies elegidas y utilizadas en varias ciudades de la provincia de Jujuy para formar parte del arbolado público (Martínez 2016). El naranjo es una especie introducida de origen asiático, naturalizada en la Argentina en el NOA y en el Noreste del país (NEA). El fruto se usa para elaborar dulces y licores; su esencia, para perfumería y con fines medicinales; muy utilizado en el arbolado de calles (Romeo and Entrocassi 2012).

En cada ciudad se seleccionaron dos ambientes según el grado de urbanización: centro (urbano) y borde (suburbano). En cada sitio se muestrearon tres árboles por especie. Los hemípteros se colectaron mediante dos técnicas: a) aspirado del follaje durante 15 minutos por cada árbol, con un aspirador Stihl SH-86C adaptado, y b) extracción al azar de 10 ramas/árbol. Las muestras obtenidas de los aspirados y las ramas fueron individualizadas en bolsas de voile y plásticas, respectivamente, etiquetadas y llevadas al laboratorio para su posterior estudio.

Los ejemplares de Hemiptera obtenidos fueron individualizados y cuantificados bajo microscopio estereoscópico y acondicionados en frascos con alcohol al 70\%. En algunos casos se realizaron preparaciones en seco o microscópicas para facilitar su identificación, hasta el menor nivel taxonómico posible, utilizando claves específicas para cada grupo. Se completó el registro de morfoespecies encontradas con información bioecológica y la información obtenida fue volcada en tablas y bases de datos para su posterior análisis.

\section{Análisis de datos}

Se confeccionaron tablas comparativas de abundancia, riqueza específica y diversidad de Shannon (Villarreal et al. 2004) de las morfoespecies de Hemiptera para cada sitio de estudio. Se calcularon los intervalos de confianza para los índices de diversidad de Shannon mediante la técnica de Bootstrap (Pla 2004) usando el software Infostat. Se compararon las abundancias, riqueza y diversidad entre los tres subórdenes en función de las especies arbóreas, las localidades, la estacionalidad y el grado de urbanización. Esto permitió caracterizar la diversidad de hemípteros asociados a estas tres especies del arbolado público en las tres localidades estudiadas en la provincia de Jujuy.

Con el objeto de observar las especies compartidas y exclusivas se representó, mediante un diagrama de Venn, la composición de Hemiptera en función de la especie arbórea hospedante. Se determinó la dominancia de las morfoespecies para cada especie arbórea, considerando a cada una de ellas como dominante cuando presenta una abundancia relativa superior a $1 / S$, donde $S$ es el número total de morfoespecies registrada en cada especie de árbol. Los cambios en la abundancia y dominancia de hemípteros fueron analizados mediante curvas de rango de abundancia realizadas por medio del software Excel 2013. Este análisis permitió observar cambios en la presencia y abundancia de hemípteros en cada especie arbórea (Feinsinger 2001).

\section{Resultados}

Se obtuvo un total de 4339 individuos de Hemiptera pertenecientes a los subórdenes Auchenorrhyncha (8 familias, 30 morfoespecies y 1044 individuos), Heteroptera (8 familias, 20 morfoespecies y 196 individuos) y Sternorrhyncha ( 9 familias, 33 morfoespecies y 3099 individuos). De las 83 morfoespecies 
Tabla 1. Morfoespecies de hemípteros registradas en el arbolado público de la provincia de Jujuy. Referencias para árboles: $\mathrm{L}=$ lapacho, $\mathrm{N}=$ naranjo, $\mathrm{P}=$ pacará, ${ }^{*}=$ dominantes; para localidades: $\mathrm{EC}=\mathrm{El}$ Carmen, $\mathrm{SP}=\mathrm{San}$ Pedro, SS=San Salvador de Jujuy.

Table 1. Hemiptera morphospecies registered in the public trees of the province of Jujuy. References for trees: $\mathrm{L}=$ lapacho, $\mathrm{N}=$ orange, $\mathrm{P}=$ pacará, ${ }^{*}=$ dominant; for localities: $\mathrm{EC}=\mathrm{El}$ Carmen, $\mathrm{SP}=\mathrm{San}$ Pedro, $\mathrm{SS}=$ San Salvador de Jujuy.

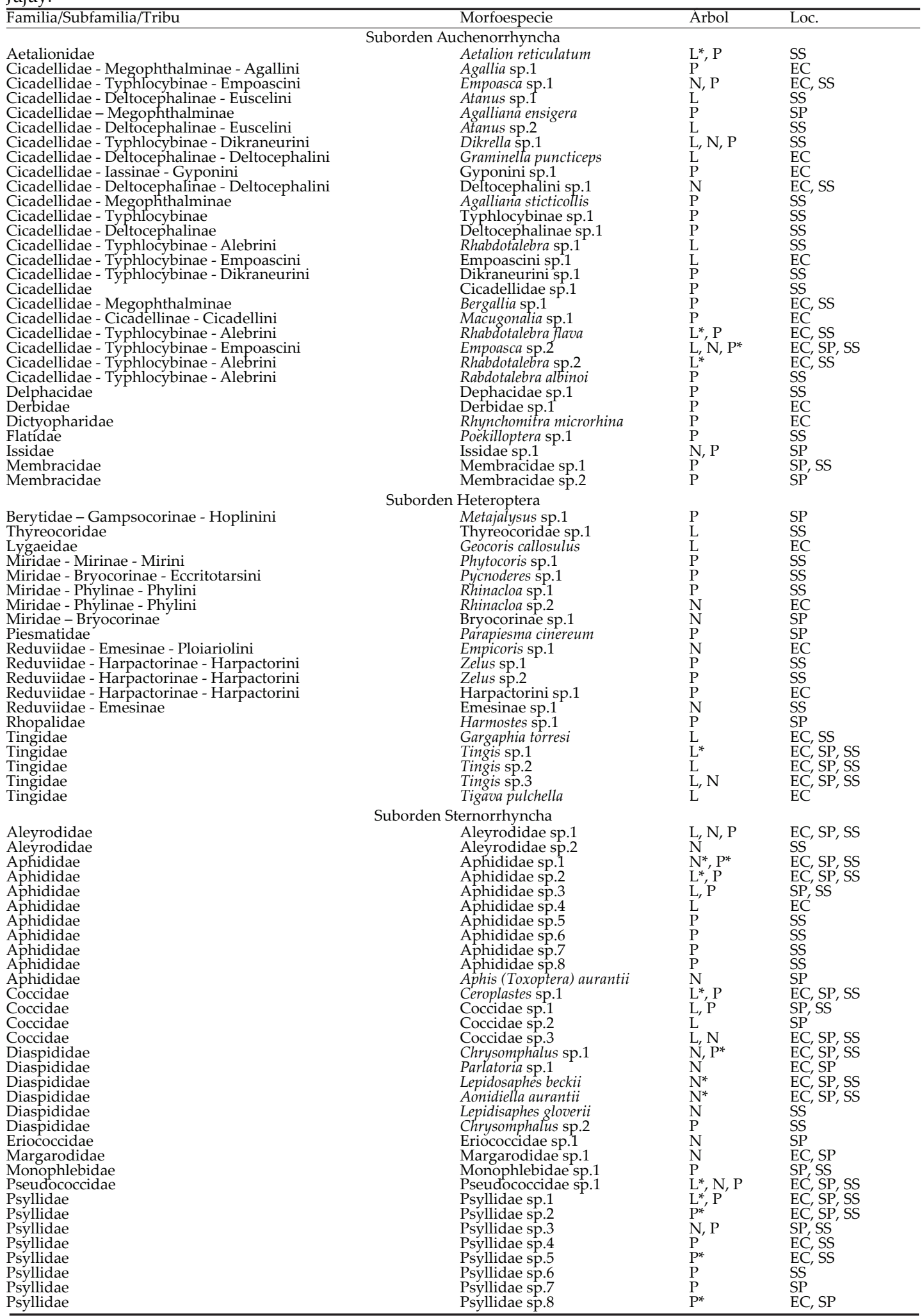


registradas, 15 fueron identificadas a nivel de especie, 27 a nivel de género, 5 a nivel de tribu, 4 a nivel de subfamilia y el resto a nivel de familia (Tabla 1).

Se cita por primera vez para la provincia de Jujuy a Rhabdotalebra albinoi Paradell y Catalano y a Rhabdotalebra flava Catalano (Cicadellidae: Typhlocybinae: Alebrini) sobre pacará, y a R. flava y al género Tingis Fabricius sobre H. impetiginosus (lapacho rosado) como especie hospedera.

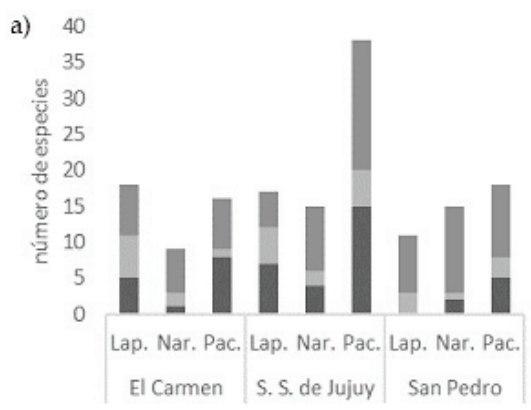

a Auchenorrhyncha $\mathbf{n}$ Heteroptera $\mathbf{m}$ Sternorrhyncha

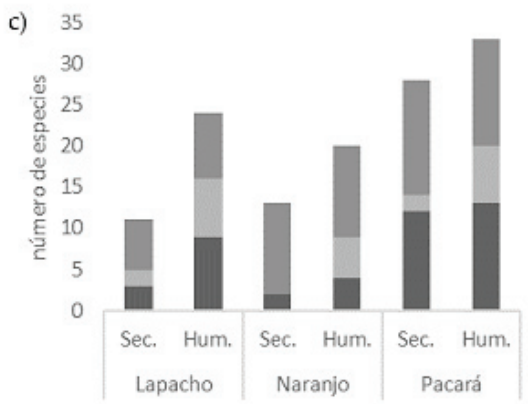

- Auchenorrhyncha $\mathbf{n}$ Heteroptera $\mathbf{n}$ Stemorrhyncha

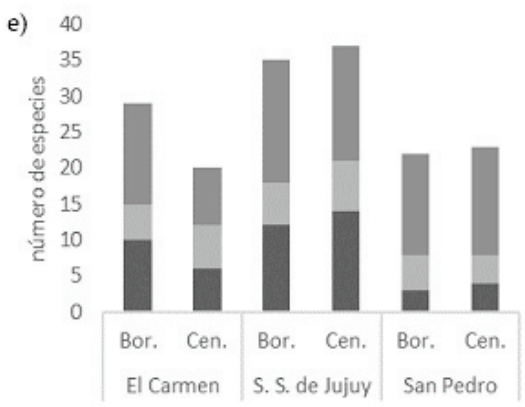

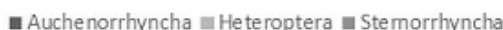

Respecto al hábito alimentario, Auchenorrhyncha y Sternorrhyncha son fitófagos, al igual que algunas familias de Heteroptera como Berytidae, Thyreocoridae, Piesmatidae, Rhopalidae, Tingidae y algunas morfoespecies de Miridae (Pycnoderes sp.1 y Bryocorinae sp.1). Entre las especies depredadoras se encontraron Geocoris callosulus Berg, 1878, las cinco morfoespecies de la familia Reduviidae y una morfoespecie de la familia Miridae (Phytocoris sp.1). De esta última familia también se registraron

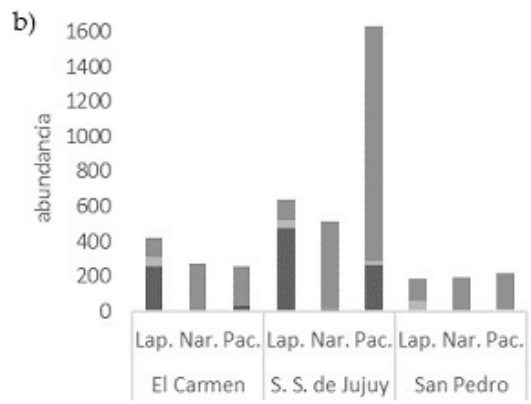

- Auchenorrhyncha a Heteroptera $\mathbf{E}$ Sternorrhyncha

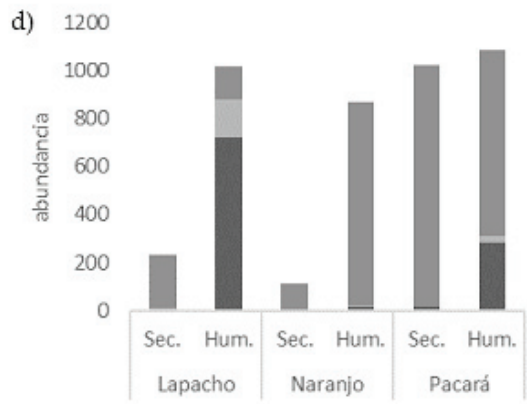

- Auchenorrhyncha $=$ Heteroptera $\|$ Sternorrhyncha

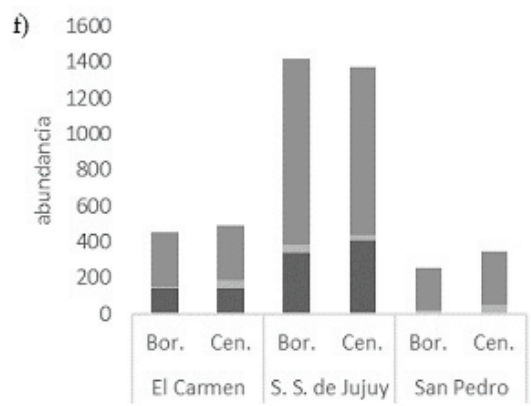

- Auchenorrhyncha $\mathbf{m}$ Heteroptera $\mathbf{n}$ Sternorrhyncha

Figura 2. Riqueza (a, c, e) y abundancia (b, d, f) de hemípteros en el arbolado público de S. S. de Jujuy, El Carmen y San Pedro durante setiembre-octubre de 2016 y febrero-marzo de 2017. En a y b se muestran los valores observados en cada especie arbórea (Lap.: lapacho, Nar.: naranjo, Pac.: pacará) y discriminados según localidad; en c y d, los valores de cada especie arbórea discriminados por estación (Sec.: seca, Hum.: Húmeda); en e y f, los valores obtenidos en cada localidad discriminados por ambiente (Bor.: borde, Cen.: centro).

Figure 2. Hemiptera richness (a, c, e) and abundance (b, d, f) in the public trees from S. S. de Jujuy, El Carmen and San Pedro during September-October 2016 and February-March 2017. In a and b, values observed in each tree species (Lap.: lapacho, Nar.: orange, Pac.: pacará) and discriminated according localities; in c and d, values of each tree species discriminated by season (Sec.: dry, Hum.: Wet); in e and f, values obtained in each locality discriminated by environment (Bor.: border, Cen.: center). 
dos ejemplares de dieta mixta: Rhinacloa sp.1 y Rhinacloa sp.2.

\section{Riqueza y abundancia en relación con la localidad y la especie arbórea}

San Salvador de Jujuy fue la localidad con mayor riqueza de especies y abundancia (56 morfoespecies, 28 exclusivas, 2790 ejemplares), mientras que El Carmen (39 morfoespecies, 13 exclusivas) y San Pedro (34 morfoespecies, 11 exclusivas) presentaron riquezas similares, pero difirieron en sus abundancias (951 y 598 ejemplares respectivamente) (Figura $2 \mathrm{a}$ y b). En la localidad de San Salvador de Jujuy, el pacará fue la especie que presentó los mayores registros de riqueza y abundancia; en El Carmen lo fue el lapacho, y en San Pedro, la riqueza y abundancia fueron similares para las tres especies de árboles estudiadas.

En las tres localidades y especies de árboles, el suborden mejor representado, tanto en riqueza como en abundancia, fue Sternorrhyncha. Para Auchenorrhyncha, la mayor riqueza se encontró en pacará (15) y la mayor abundancia en lapacho (472), ambas en San Salvador de Jujuy. Heteroptera alcanzó la mayor riqueza (6) en El Carmen sobre lapacho mientras que la abundancia fue similar entre lapachos de las localidades de San Pedro (57) y El Carmen (55).

\section{Riqueza y abundancia en relación a la estacionalidad}

En las tres especies arbóreas, tanto en la estación seca como en la húmeda, el suborden Sternorrhyncha fue el más abundante (1342 y 1757 , respectivamente) y rico en morfoespecies (31 y 32, respectivamente). La excepción se registró en lapacho durante la estación húmeda, donde los Auchenorrhyncha fueron los más abundantes, seguidos por los Heteroptera. Auchenorrhyncha alcanzó la mayor abundancia (1024) y riqueza (26) en la estación húmeda, así como también los Heteroptera mayor abundancia (188) y riqueza de especies (19) (Figura 2 c y d).

\section{Riqueza y abundancia según el grado de urbanización y localidad}

En general, no se observaron diferencias de riquezas según el grado de urbanización y las localidades estudiadas. El Carmen fue la única localidad que mostró mayor diferencia en la riqueza de Auchenorrhyncha y Sternorrhyncha, registrando 10 y 14 morfoespecies respectivamente en el sector periurbano (borde) y 6 y 8 morfoespecies, respectivamente, en el sector urbano (centro). Con respecto a la abundancia, la mayor diferencia se observó en Heteroptera en la localidad de El Carmen, registrando 9 ejemplares en el sector periurbano y 50 en el urbano (Figura 2 e y f).

\section{Diversidad}

Se encontraron diferencias significativas sólo en Auchenorrhyncha. Los mayores valores se registraron en pacará $(\mathrm{H}: 4.44 ; P<0.05 ; \mathrm{n}=36)$ (Figura 3).

\section{Composición de morfoespecies en relación a las especies arbóreas}

El pacará es la especie arbórea con mayor número de morfoespecies (54) y morfoespecies exclusivas (38). En orden de importancia le siguen el lapacho con 27 morfoespecies (14 exclusivas) y el naranjo con 24 morfoespecies (13 exclusivas). Las morfoespecies compartidas por los tres árboles hospedantes fueron Dikrella sp.1, Empoasca sp.2, Aleyrodidae sp.1 y Pseudococcidae sp.1 (Figura 4).

\section{Dominancia de morfoespecies}

En pacará se registraron tres morfoespecies dominantes, de las cuales Psyllidae sp.2, exclusiva de este árbol, y Aphididae sp.1 pertenecen al suborden Sternorrhyncha,

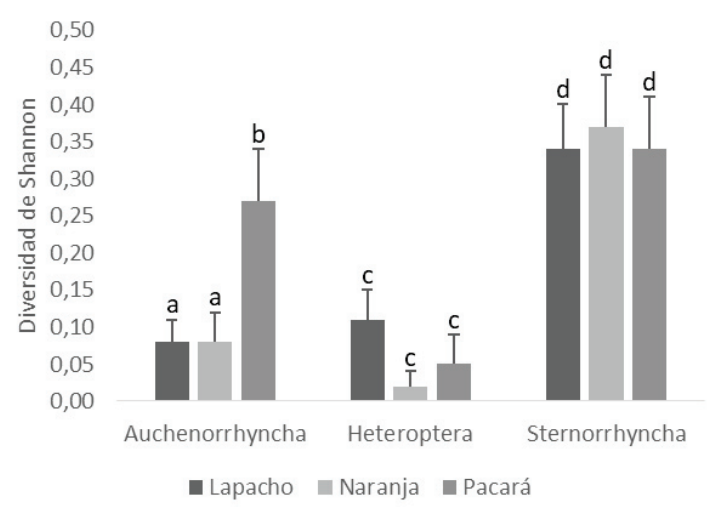

Figura 3. Diversidad de hemípteros en el arbolado público durante setiembre-octubre de 2016 y febrero-marzo de 2017. Las barras verticales indican errores estándar y las letras sobre las mismas indican diferencias significativas según la prueba de Kruskal-Wallis $(P<0.05)$.

Figure 3. Hemiptera diversity of public trees during September-October 2016 and February-March 2017. Standard errors are indicated with vertical bars and the letters on them indicate significant differences according to the Kruskal-Wallis test $(P<0.05)$. 


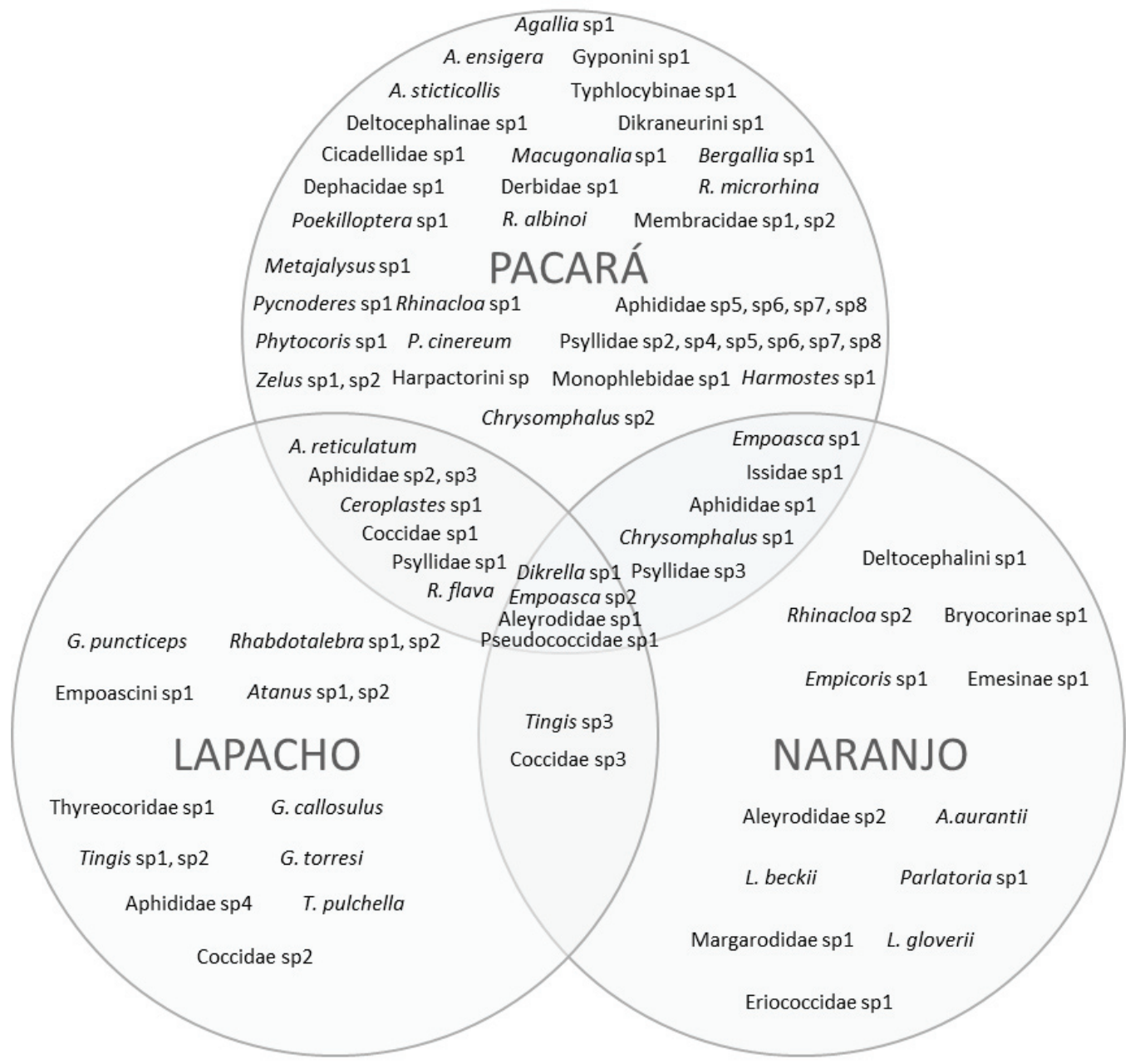

Figura 4. Diagrama de Venn mostrando la composición de morfoespecies de Hemiptera en relación con las especies arbóreas durante setiembre-octubre de 2016 y febrero-marzo de 2017.

Figure 4. Venn diagram indicating the morphospecies composition of Hemiptera in relation to tree species during September-October 2016 and February-March 2017.

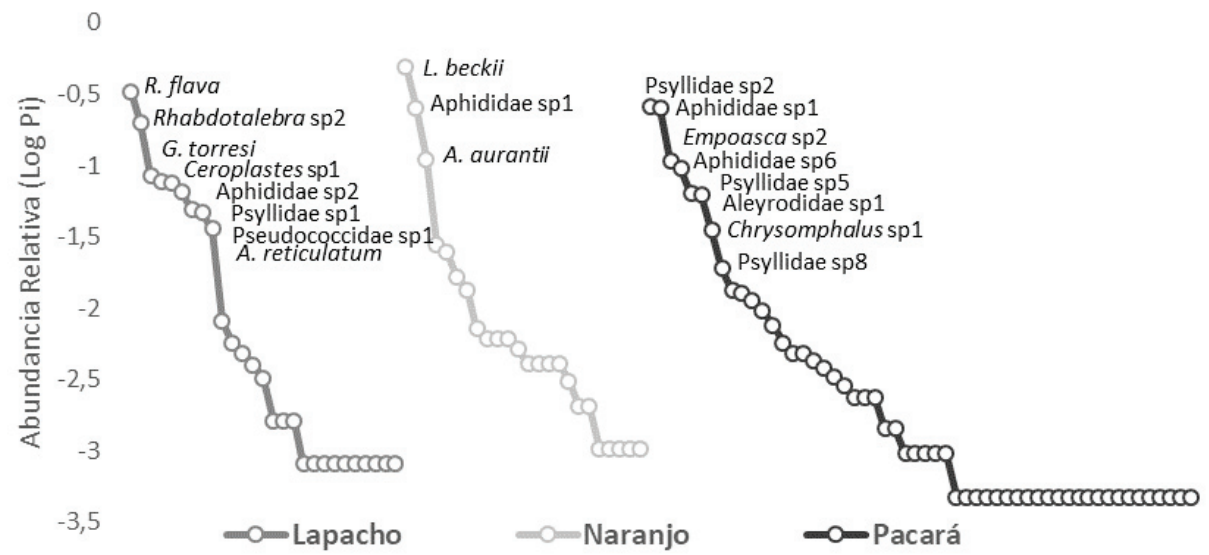

Figura 5. Curva de rango-abundancia para las morfoespecies de Hemiptera en el arbolado público durante setiembreoctubre de 2016 y febrero-marzo de 2017.

Figure 5. Range-abundance curve for Hemiptera morphospecies in public trees during September-October 2016 and February-March 2017. 
mientras que Empoasca sp.2 corresponde al suborden Auchenorrhyncha, familia Cicadellidae. En lapacho, las morfoespecies dominantes fueron Rabdotalebra flava y Rabdotalebra sp.2, del suborden Auchenorrhyncha, familia Cicadellidae, y Tingis sp.1, del suborden Heteroptera, familia Tingidae. Además, esta familia es exclusiva de este árbol hospedador. En naranjo se encontraron como dominantes y exclusivas a dos cochinillas de la familia Diaspididae (Lepidosaphes beckii Newman y Aonidiella aurantii Maskell y sólo como dominante a una especie de la familia Aphididae: Aphididae sp.1; todas pertenecientes al suborden Sternorrhyncha (Figura 5).

\section{Discusión}

La mayor riqueza y abundancia de hemípteros asociados a las especies arbóreas estudiadas en las tres localidades estuvo dada por las morfoespecies de Sternorrhyncha pertenecientes a las familias Aphididae y Psyllidaeen pacará,Coccidaey Pseudococcidae en lapacho y Diaspididae en naranjo. Esto podría deberse a su comportamiento gregario y a la capacidad de formar grandes colonias en distintas partes de las plantas (Grazia et al. 2012).-

Muchas especies de Auchenorrhyncha son indicadoras botánicas, dado que prefieren vegetales específicos como alimento y otros como sustrato para la oviposición (Nielson and Knight 2000). En este trabajo se puede observar que existe una tendencia a la especificidad entre el fitófago y el hospedador, ya que la mayoría de los auquenorrincos encontrados son específicos para una de las especies arbóreas estudiadas. Rabdotalebra flava y Rabdotalebra sp.2 son propias del lapacho, hospedador primario, ya que cumplen su ciclo de vida allí. Otras, como las especies del género Empoasca y Dikrella sp.1, son generalistas y oportunistas, ya que se las encontró tanto en lapacho como pacará y naranjo (hospedadores secundarios), utilizándolos sólo para alimentarse. Este tipo de comportamiento favorece la adquisición, el transporte y la transmisión de patógenos a las plantas (fitoplasmas, espiroplasmas y micoplasmas) y, en consecuencia, pueden transformarse en plagas (Paradell and Cavichioli 2014).

De acuerdo con los resultados obtenidos de riqueza y abundancia en función de las localidades estudiadas, San Pedro fue la que presentó menor riqueza y abundancia. Según
Hidalgo-Gato et al. (2012), las comunidades de Auchenorrhyncha se ven afectadas por las características ambientales particulares de cada localidad. Teniendo en cuenta que San Pedro es una ciudad agroindustrial con características ambientales propias de esta actividad, es esperable que la riqueza y la abundancia de los auquenorrincos sea menor con respecto a San Salvador de Jujuy y El Carmen. Sería necesario corroborar estos resultados realizando estudios específicos referidos a la influencia de los factores ambientales sobre estos indicadores.

En este estudio, Heteroptera fue el suborden menos representado del total de hemípteros registrados. Sin embargo, los trabajos realizados por Bolger et al. (2000) y Helden y Leather (2004) reportaron la presencia de heterópteros en zonas urbanas, y lo muestran como un grupo relativamente abundante y diverso. Sería conveniente profundizar el relevamiento para determinar cuáles podrían ser las causas por la que es el grupo menos representado en este trabajo, ya sea ampliando el listado de árboles hospederos o intensificando los muestreos.

En cuanto a los hábitos alimentarios del suborden Heteroptera, se encontraron mayormente especies fitófagas. Las depredadoras, como las pertenecientes a las familias Geocoridae, Reduviidae y algunas especies de Miridae, se registraron en menor cantidad. De la Mora-Estrada et al. (2017) establece que las especies fitófagas son abundantes debido a la disponibilidad de los recursos alimenticios y que, a su vez, estos fitófagos pueden ser una fuente importante de alimento de los depredadores.

La estacionalidad es un factor que podría afectar la riqueza y la abundancia de los Auchenorrhyncha y Heteroptera, y no la de Sternorrhyncha, ya que no se observan diferencias en cuanto a la riqueza, pero sí en la abundancia según la especie hospedadora. Dentro de este suborden, las familias Coccidae y Diaspididae se destacaron por su presencia continua a lo largo del año. Esto podría explicarse si se tienen en cuenta las características biológicas y ecológicas de estos insectos. El cuerpo de las hembras se encuentra muy modificado en relación a la vida parasitaria (Claps 1990; Zamar and Claps 2003). Presentan dificultad de dispersión ya que su transporte es pasivo (i.e., viento, pájaros, insectos $u$ otros animales) y la mayor dispersión ocurre por la actividad humana (Claps and dos Santos Wolff 2014). 
En relación con el grado de urbanización (urbano y suburbano), para el suborden Auchenorrhyncha no se observó un patrón que indique diferencias en cuanto a la riqueza y abundancia. Según Nielson y Knight (2000) y Landero-Torres et al. (2011), la distribución de algunos Auchenorrhyncha (como es el caso de Cicadellidae, que presenta una distribución cosmopolita) ocupa diversos nichos ecológicos y se alimenta de una amplia variedad de plantas. La dependencia de sus especies por un determinado huésped (i.e., por alimento, protección y perpetuación) es uno de los mecanismos más importantes que determina la distribución de este grupo. Para los subórdenes Heteroptera y Sternorrhyncha tampoco se observó un patrón que pueda estar afectando su distribución.

De las principales especies dominantes del lapacho, Rabdotalebra flava y Rabdotalebra sp.2 (Auchenorrhyncha: Cicadellidae: Typhlocybinae), Rabdotalebra flava fue citada por primera vez en este hospedero por Catalano et al. (2010) para la provincia de Tucumán. El género Tingis Fabricius incluye numerosas especies cuyas plantas hospedadoras pertenecen a Bignoniaceae, Dileneaceae y Malpighiaceae (Drake and Ruhoff 1965). Tingis fue registrado en lapacho (familia Bignoniaceae) y en menor abundancia en naranjo (familia Rutaceae). Otra de las especies dominantes en lapacho es Ceroplastes sp.1 (Sternorryncha: Coccidae), que fue registrada principalmente en árboles ubicados en calles y avenidas de mayor tránsito vehicular lo que, probablemente, favorecería su dispersión por este tipo de disturbio de origen antrópico.

En naranjo se destacaron las cochinillas Diaspididae (Lepidosaphes beckii y Aonidiella aurantii) y el pulgón Aphididae sp.1. Las dos especies de cochinillas, que coexisten en el naranjo, son propias de árboles frutales y ornamentales, y por las características de su cubierta protectora pueden vivir en condiciones extremas como por ejemplo aire contaminado o enrarecido (Claps and dos Santos Wolff 2014).

De las tres especies arbóreas estudiadas en este trabajo, el pacará es la que se destaca por tener la mayor riqueza y abundancia de Hemípteros de la familia Aleyrodidae y Psyllidae. Según Grazia et al. (2012), la familia Aleyrodidae es considerada una plaga clave de cítricos y otros cultivos de interés agrícola. Al presente no se registraron estudios sobre esta familia asociada a árboles de ambientes urbanos en la provincia de Jujuy. La presencia de estos insectos y sus hábitos polífagos nos permiten inferir que los aleiródidos no sólo prefieren plantas de interés agrícola, sino también especies vegetales nativas que revisten otro interés para el ser humano, como lo es el arbolado público. Las morfoespecies Aleyrodidae sp.1 y Psyllidae sp.2 se destacaron por su dominancia en pacará. Esta última fue también registrada sobre otras especies arbóreas de la familia Fabaceae utilizadas en arbolado público (Magistrali et al. 2009).

Las interacciones ecológicas entre plantas y animales constituyen la base del funcionamiento de los ecosistemas (Loera-Padilla et al. 2015). La información proporcionada contribuye al entendimiento de la función y la estructura de los ecosistemas urbanos desde el enfoque de la interacción planta-hemíptera, y son datos de base para evaluar la dinámica de hemípteros asociados al arbolado público de la provincia de Jujuy. Sería propicio, en futuros estudios, indagar acerca de la influencia de los factores que inciden sobre la dinámica de la comunidad de Hemiptera a través de muestreos específicos diseñados para evaluar el comportamiento de los tres subórdenes que lo componen.

Agradecimientos. Este trabajo fue realizado con fondos otorgados por la SECTER-UNJu (Proyectos F/C004 y F/B005). Se agradece por la colaboración en la identificación del material a L. E. Claps, M. I. Catalano, E. Guilbert y S. I. Montemayor, y a M. I. Zamar por el asesoramiento y lectura crítica del manuscrito.

\section{REFERENCIAS}

Agostini de Manero, E., and S. Muruaga De L`Argentier. 1987. Catálogo de organismos animales perjudiciales en cultivos del noroeste argentino. Ciencias Agrarias 5:1-40.

Biloni, J. S. 1990. Árboles autóctonos argentinos. Tipográfica Editora Argentina, Buenos Aires. Pp. 335. ISBN: 950521-069-8.

Bolger, D. T., A. V. Suárez, K. V. Crooks, S. A. Morrison, and J. T. Case. 2000. Arthropods in urban habit fragments in Southern California: area, age, and edge effects. Ecological Applications 4:1230-1248. https://doi.org/10.1890/10510761(2000)010[1230:AIUHFI]2.0.CO;2.

Brown, A., U. Martínez Ortiz, M. Acerbi, and J. Corcuera. 2005. La situación ambiental Argentina 2005. Fundación vida Silvestre Argentina, Buenos Aires. Pp. 577. 
Cabrera, A. L. 1976. Regiones Fitogeográficas Argentinas. Enciclopedia Argentina de Agricultura y Jardinería. Fascículo 1, Tomo II, Segunda Edición. Editorial ACME.

Carver, M., G. F. Gross, and T. E. Woodward. 1991. Pp. 429-509 en The Insects of Australia I (Cap. 30). Commonwealth Scientific and Industrial Research Organization. Australia. Division Entomology.

Catalano, M. I., S. L. Paradell, and A. M. M. De Remes Lenicov. 2010. Revision of the genus Rhabdotalebra Young (Hemiptera: Cicadellidae: Thyphlocibinae: Alebrini) and description of two new species from Argentina. Zootaxa 2602:53-60. https://doi.org/10.11646/zootaxa.2601.1.5.

Catalano, M. I., S. L. Paradell, and C. H. Dietrich. 2013c. First report on the leafthopper genus Balera Young (Hemiptera, Cicadellidae, Typhlocybinae, Alebrini) from Argentina, and description of a new species. Zookeys 352:1-7. https: //doi.org/10.3897/zookeys.352.6283.

Catalano. M. I., S. L. Paradell, and C. H. Dietrich. 2013a. Four new species of genus Parallaxis McAtee (Hemiptera: Cicadellidae: Typhlocybinae: Dikraneurini) from Argentina, with a key to known species. Zootaxa 3737(3):241-250. ISSN: 1175-5326. https://doi.org/10.11646/zootaxa.3737.3.3.

Catalano. M. I., S. L. Paradell, and C. H. Dietrich. 2013b. Three news species of Neozygina Dietrich and Dimitriev (Hemiptera: Cicadellidae: Typhlocybinae) from Argentina, with a key to South- American species. Zootaxa 3652(1): 156-162. ISSN: 1175-5326. https://doi.org/10.11646/zootaxa.3652.1.5.

Claps, L. E. 1990. Características del ciclo biológico de Cornuaspis beckii (Newman, 1869) e Insulaspis gloverii (Packar, 1869) en condiciones de insectario (Insecta; Cocoidea; Diaspididae). CIRPON, Revista de Investigación 5(1):7-16.

Claps, L. E., and V. R. Dos Santos Wolff. 2014. Hemiptera - Diaspididae. Pp. 293-302 en L. E. Claps, J. Morrone and S. Roig-Juñent (eds.). Biodiversidad de Artrópodos de Argentinos, volumen 3. Editorial INSUE-UNT.

Claps, L. E., G. Debandi, and S. Roig-Juñent. 2008. Biodiversidad de Artrópodos Argentinos, vol. 2. Editorial Sociedad Entomológica Argentina, Mendoza. Pp. 615.

Contreras, E. F., and M. C. Cosacrón. 2012. The Aradidae (Insecta, Hemiptera, Heteroptera) of Argentina. Zootaxa 3500:1-35. ISSN: 1175-5334. https://doi.org/10.11646/zootaxa.3500.1.1.

Coscarón, M. C. 2017. A catalogue of the Heteroptera (Hemiptera) or the true bugs of Argentina. Zootaxa 4295:432. https://doi.org/10.11646/zootaxa.4295.1.1.

De la Mora-Estrada, L. F., L. Ruiz-Montoya, N. Ramírez-Marcial, A. Morón-Ríos, and M. C. Mayorga-Martínez. 2017. Diversidad de chinches (Hemiptera: Heteroptera) en bosques secundarios de pino-encino de San Cristobal de Las Casas, Chiapas, México. Revista Mexicana de Biodiversidad 88:86-105. https://doi.org/10.1016/j.rmb.2017.01.016.

Dellapé, G. 2015. Synopsis of the Acanthosomatidae (Heteroptera) from Argentina. Revista de la Sociedad Entomológica Argentina 75:81-90.

Dellapé, G., D. A. Rider, and P. M. Dellapé. 2015. Notes on Distributions for Argentinian Pentatomidae (Heteroptera: Pentatomoidea), with new records in the country. Revista Brasileira de Entomologia 59:169-176. https://doi.org/ 10.1016/j.rbe.2015.06.001.

Dellapé, G., S. Paradell, L. Semorille, and L. Delfederico. 2016. Potential vectors of Xilella fastidiosa: a study of leafhoppers in citrus agrosystems affected by Citrus Variegated Chlorosis. Entomologia Experimentais et Applicata 161:92-103. https://doi.org/10.1111/eea.12491.

Drake, C. J., and F. A. Ruhoff. 1965. Lacebugs of the World: a catalog (Hemiptera: Tingidae). Washington D.C., Smithsonian Institution. Pp. 634. https://doi.org/10.5479/si.03629236.243.1.

Erwin, T. L. 1982. Tropical forests: their richness in Coleoptera and other arthropod species. The Coleopterists Bulletin 36:74-75.

Feinsinger, P. 2001. Designing field studies for biodiversity conservation. Island Press.

Gaston, K. J. 1991. The magnitude of global insect species richness. Conservation Biology 5(3):283-296. https://doi.org/ 10.1111/j.1523-1739.1991.tb00140.x.

Grazia, J., R. R. Cavichioli, V. R. S. Wolff, J. A. M. Fernandes, and D. A. Takiya. 2012. Hemiptera. Pp. 347-405 in J. A. Rafael, G. A. R. Melo, C. J. B. Carbalho and S. Casari (orgs.). Os Insectos do Brazil: Diversidad e Taxonomia. Cap. 28.

Hamity, V. C., V. Curzel, N. Zapana, F. Fernández, F. Guzmán, N. Bejarano, and L. Conzi. 2016. Estudio preliminar del amarillamiento del duraznero en Jujuy. Libro de resúmenes de las X Jornadas Científico-técnicas de la Facultad de Ciencias Agrarias. Trabajo completo. Pp.: 80-87. ISBN: 978-987-3926-20-4.

Helden, A. J., and S. R. Leather. 2004. Biodiversity on urban roundabout-Hemiptera, management and the species-area relationship. Basic and Applied Ecology 5:367-377. https://doi.org/10.1016/j.baae.2004.06.004.

Hidalgo-Gato, M. M., R. Rodríguez-León, and N. Ricardo. 2012. Estimación de la riqueza de especies y abundancia de Auchenorrhyncha (Insecta: Hemiptera) presente en bosque semideciduo y vegetación sinantrópica de tres localidades de la Sierra del Rosario, Cuba. Boletín de la Sociedad Entomológica Aragonesa 50:481-493.

Landero-Torres, I., E. Blanco Rodríguez, J. Ramos-Elorduy, M. Herrera Tenorio, H. Oliva Rivera, and M. E. GalindoTovar. 2011. Diversidad de la familia Cicadellidae (Hemiptera) en la congregación del Barreal, municipio de Córdoba Ver. Entomología Mexicana 10:202-205.

Linares, M. A., and L. E. Neder. 1999. Presencia de Pterygogramma membraciphagum Viggiani, 1992 (Hymenoptera: Trichogrammatidae), enemigo natural de Guayaquila sp. (Homoptera: Membracidae) en la provincia de Jujuy. Neotropica 45(113-114):118.

Linares, M. A., L. E Neder, and C. Dietrich. 2010. Description of immature stages and life cycle of the treehopper, Guayaquila projecta. Journal of Insect Science 10(1):199. https:/ / doi.org/10.1673/031.010.19901.

Loera-Padilla, F. J., E. C. López-Barboza, A. González-Rodríguez, and P. Cuevas-Reyes. 2015. Variación especial de la 
comunidad de artrópodos del dosel asociados a Quercus castanea a lo largo de un gradient de humedad. Boletín de la Sociedad Mexicana de Entomología 1:47-52.

Magistrali, I. C., N. dos Anjos, R. M. Souza, and C. L. Duarte. 2009. Ocorrência e infestação de Euphalerus clitoriae Burckhardt and Guajará, 2000 (Hemiptera: Psylloidea) em árvores de sombreiro (Clitoria fairchildiana Howard) utilizadas na arborização urbana de Viçosa-MG. Revista da Sociedade Brasileira de Arborização Urbana 4(4):100110. https:/ /doi.org/10.5380/revsbau.v4i4.66444.

Martínez, S. M. 2016. Guía de Árboles Nativos del Noroeste Argentino. 1era. Ed. Ampliada. Editor Tonda Martínez, S. E. ISBN 978-987-42-0581-0, Salta, Argentina. Pp. 200.

McIntyre, N. E. 2000. Ecology of urban arthropods: a review and a call to action. Annals of the Entomological Society of America 93(4):825-835. https:/ / doi.org/10.1603/0013-8746(2000)093[0825:EOUAAR]2.0.CO;2.

McKinney, M. L. 2002. Urbanization, biodiversity, and conservation. Bioscience 52:883-890. https://doi.org/10.1641/ 0006-3568(2002)052[0883:UBAC]2.0.CO;2.

Melo, M. C., and P. M. Dellapé. 2013. Catalogue of the Pyrrhocoroidea (Heteroptera) of Argentina. Revista de la Sociedad Entomológica Argentina 72(1-2): 55-74.

Morrone, J. J., and S. Coscarón. 1998. Biodiversidad de artrópodos argentinos: una perspectiva biotaxonómica. Ediciones Sur: Buenos Aires, Argentina. Pp. 599.

Muruaga De L'Argentier, S., and E. Agostini. 1995. Contribución al estudio de las especies de Cicadellidae (Homoptera) presentes en el cultivo de poroto en Jujuy (Argentina). Revista de la Sociedad Entomológica Argentina 54(1-4):7782.

Nielson, M. W., and W. J. Knight. 2000. Distributional patterns and possible origin of leafhhoppers (Homoptera: Cicadellidae). Revista Brasileira de Zoologia 17:81-156. https://doi.org/10.1590/S0101-81752000000400004. https:// doi.org/10.1590/S0101-81752000000100010.

Paradell, S., and R. Cavichioli. 2014. Cicadellidae. Pp. 319-334 en S. Roig-Juñent, L. E. Claps and J. J. Morrone (directores). 2014. Biodiversidad de Artrópodos Argentinos, volumen 3. Editorial INSUE-UNT, San Miguel de Tucumán, Argentina.

Pla, L. 2004. Bootstrap confidence intervals for the Shannon biodiversity index: a simulation study. Journal of Agricultural, Biological, and Environmental Statistics 9(1):42-56. https:/ /doi.org/10.1198/1085711043136.

Rebele, F. 1994. Urban ecology and special features of urban ecosystems. Global Ecology and Biogeography Letters 4 : 173-187. https://doi.org/10.2307/2997649.

Roig-Juñent, S., L. E. Claps, and J. J. Morrone. 2014. Biodiversidad de Artrópodos Argentinos. Volumen 3. Editorial INSUE - UNT, San Miguel de Tucumán, Argentina.

Romeo, R. A., and G. S. Entrocassi. 2012. Los Árboles de la Ciudad de San Salvador de Jujuy. Provincia de Jujuy, Argentina, primera parte. Universidad Nacional de Jujuy, EdiUnju. Pp. 183. ISBN: 13:978-950-721-370-0.

Schuh, R. T., and J. A. Slater. 1995. True bugs of the Word (Hemiptera: Heteroptera). Classification and Natural History. Ithaca: Cornell University Press.

Stork, N. E. 1988. Insect diversity: facts, fiction and speculation. Biological Journal of the Linnean Society 35(4):321-337. https://doi.org/10.1111/j.1095-8312.1988.tb00474.x.

Villarreal, H., M. Álvarez, S. Córdoba, F. Escobar, G. Fagua, F. Gast, H. Mendoza, M. Ospina, and A. M. Umana. 2004. Manual de métodos para el desarrollo de inventarios de biodiversidad. Programa inventarios de Biodiversidad. Instituto de Investigación de Recursos Biológicos Alexander von Humboldt. Bogotá, Colombia. Pp. 236.

Wilson, E. O. 1985. The biological diversity crisis. Bioscience 35:700-706. https://doi.org/10.2307/1310051.

Zamar, M. I., and L. E. Claps. 2003. Morfología de los estados inmaduros y adulto de Pinnaspis aspidistrae (Hemiptera: Diaspididae), con notas sobre su biología. Revista de la Sociedad Entomológica Argentina 62(1-2):35-42. 